\title{
Esfuerzo de corte en Interfaces $\mathrm{Fe} / \mathrm{Fe}_{3} \mathrm{O}_{4}$
}

\author{
Shear Behavior of $\mathrm{Fe} / \mathrm{Fe}_{3} \mathrm{O}_{4}$ interfaces
}

\author{
Mariano Forti ${ }^{1,2}$, Paula Alonso ${ }^{1,2}$, Pablo Gargano ${ }^{1,2}$, \\ Gerardo Rubiolo ${ }^{1,2,3}$
}

\author{
${ }^{1}$ Comisión Nacional de Energía Atómica, Gerencia de Área Materiales. Centro Atómico Constituyentes, Avenida Gene- \\ ral Paz 1499 (1650), Buenos Aires, Buenos Aires, Argentina. \\ e-mail: mforti@cnea.gov.ar \\ ${ }^{2}$ Instituto Sabato, Universidad Nacional de General San Martin, San Martin, Buenos Aires, Argentina. \\ 3 Consejo Nacional de Investigaciones Científicas y Tecnológicas, CONICET, Buenos Aires, Buenos Aires, Argentina.
}

\section{RESUMEN}

La estabilidad mecánica de los óxidos formados sobre las aleaciones de uso industrial, y su adhesión al sustrato metálico es de vital importancia para determinar la susceptibilidad de las aleaciones a los medios en contacto y la aplicabilidad en condiciones de alta temperatura u otras condiciones extremas. En este contexto, la energía de adhesión es uno de los parámetros principales a determinar. Los métodos atomísticos como la Teoría del Funcional de la Densidad (DFT) se presentan como una herramienta fundamental para calcular este parámetro en interfases Oxido/Metal. En este trabajo se estudia con esta técnica la interfaz $\mathrm{Fe}(\mathrm{BCC}) /$ Magnetita. El interés en este sistema radica en que se ha visto que la magnetita $\left(\mathrm{Fe}_{3} \mathrm{O}_{4}\right)$ es el óxido en contacto con el metal en condiciones de corrosión generalizada, e incluso las capas de óxido pasivante pueden tener cierta similitud con esta desde el punto de vista estructural. Dado que la magnetita es una espinela inversa de estructura cristalina $F d \overline{3} \mathrm{~m}$ y el hierro posee una estructura BCC, se modela la interfaz $\mathrm{Fe} 3 \mathrm{O} 4[001]-\mathrm{Fe}[001]$ teniendo en cuenta que experimentalmente se observa la relación de orientaciones Fe3O4[100]||Fe[110] para la misma. A lo largo de la dirección [001] en el óxido se alternan los planos de composición $\mathrm{FeO}_{2}$ y $\mathrm{Fe}$, aunque aquí solo se trata la terminación Fe de la magnetita, ya que se ha demostrado que forma la interfaz más estable [1,2]. Se utiliza DFT para calcular el trabajo necesario para deslizar las superficies en relación a las direcciones principales de la interfaz, para luego calcular el potencial interfacial en función de las coordenadas generalizadas de la misma según el modelo de WEI y HUTCHINSON [3]. Este potencial puede ser utilizado en modelos de meso escala de la interfaz, por ejemplo para el cálculo de la tenacidad de la misma.

Palabras clave: Adhesión Interfacial, Potencial Interfacial, Magnetita, DFT.

\section{ABSTRACT}

The mechanical stability of oxides formed on metallic alloys is a key concern in the determination of component susceptibility to the medium in contact and the applicability of the alloy to high temperatures and other extreme conditions. In this context, the energy of adhesion is a key parameter. Density functional Theory (DFT) and other atomistic methods are fundamental tools in the determination of this quantity for Metal/Oxide interfaces. In this paper Fe(BCC) / Magnetite interface is assessed within the DFT approach. This system is of general interest, given that magnetite $\left(\mathrm{Fe}_{3} \mathrm{O}_{4}\right)$ is the oxide in direct contact with the metal under generalized corrosion and for a wide range of conditions. Moreover, it has been demonstrated that passive films on iron alloys exhibit similar structural properties. Magnetite is an inverse spinel with $F d \overline{3} m$ structure, while Iron has a BCC crystal structure. The interface $\mathrm{Fe}_{3} \mathrm{O}_{4}[001]-\mathrm{Fe}[001]$ is modeled. The orientation relationship experimentally observed for this interface is $\mathrm{Fe}_{3} \mathrm{O}_{4}[100] \| \mathrm{Fe}[110]$. In the (001) direction, atomic layers with compositions $\mathrm{FeO}_{2}$ and $\mathrm{Fe}$ are alternated. Here only the $\mathrm{Fe}$ termination is treated, as it has been proved that it constitutes the most stable interface [1,2]. DFT is used to calculate the work needed to relatively slide the oxide and metal surfaces in reference to the principal directions of the interface. Hence, it is possible to calculate the interface potential according to the model by WEI and HUTCHINSON [3]. This potential can be used for construction of meso scale models of the interface for a complete study of its properties. 
Keywords: Interface Adhesion, Interface Potential, Magnetite, DFT.

\section{INTRODUCCIÓN}

La integridad de la capa de óxido formada sobre las aleaciones metálicas de uso industrial es importante cuando se evalúa la aplicabilidad y resistencia de la aleación. En particular el sistema $\mathrm{Fe} / \mathrm{Fe}_{3} \mathrm{O}_{4}$ tiene una gran importancia tecnológica. Entre los óxidos que se producen cuando las aleaciones de hierro son corroídas en un ambiente oxidante, la capa más interna es magnetita u otro óxido de estructura y composición similar [46]. A modo de ejemplo se puede mencionar el caso de los reactores nucleares en las tuberías de acero al carbono donde la temperatura es alta $\left(>100{ }^{\circ} \mathrm{C}\right)$ se desarrolla un film duplex [7] que consiste con una capa interna de magnetita y una capa externa de hematita. Los residuos generados a partir de la delaminación y astillado de esta capa de óxido pueden actuar como modificadores de las condiciones de flujo del líquido refrigerante y como transportadores de material radioactivo, y se han hecho grandes esfuerzos para reducir la presencia de los mismos en el fluido circulante [8,9]. Por otro lado, en los trenes de laminación en caliente los aceros al carbono desarrollan una capa de óxido que sigue creciendo durante el enfriamiento y el enrollado. Esta capa de óxido suele componerse de una capa externa de hematita $\left(\mathrm{Fe}_{2} \mathrm{O}_{3}\right)$, una capa intermedia de magnetita, y en contacto con el metal se encuentra la wüstita que por descomposición eutéctica suele dar lugar a una cantidad considerable de magnetita en equilibrio [10]. Esta capa de óxido debe ser removida entre laminaciones subsiguientes, y en los últimos años se han estado desarrollando métodos mecánicos de decapado con el objetivo de reemplazar el decapado químico que puede causar problemas ambientales [11,12]. Para estos métodos, la capacidad de remoción de la capa de óxido está íntimamente ligada a la integridad mecánica de la misma [13].

A raíz de todos estos motivos el estudio de la integridad mecánica de la capa de óxido se ha convertido en una preocupación primaria durante las últimas décadas. Una clasificación de los modelos de fractura ha sido recolectada por SCHÜTZE [14,15] considerando como parámetros al estado de tensiones que afecta al óxido superficial y la orientación relativa entre una posible fisura y la interfaz metal / óxido. Los autores también revisan los criterios de falla asociados en cada caso. El astillado se cita como un ejemplo. Este modo de falla puede encontrarse en sistemas donde la interfaz es más débil que el óxido, y bajo tensiones de compresión. La nucleación y crecimiento de fisuras pueden originarse cuando las deformaciones alcanzan un valor crítico que puede ser calculado en función de las constantes elásticas del metal y del óxido y de las propiedades de adhesión entre ambos materiales.

Es evidente entonces que las propiedades de la interfaz metal / óxido en el sistema hierro / magnetita deben ser determinada en forma precisa. Para dar una medida cuantitativa de estas propiedades, muchos autores reportan el trabajo de separación [16-18], que se define como el trabajo reversible requerido para separar la interfaz en dos superficies libres despreciando la deformación plástica.

Experimentalmente, entre los métodos para determinar el trabajo de separación pueden encontrarse el dispositivo de torsión-balance [19], el ensayo de ampollado inverso [20], el ensayo de micro tracción que se realiza dentro de un microscopio electrónico de barrido [21,22], y el ensayo de macro tracción [17]. Sin embargo, todos estos métodos requieren cierta complejidad experimental, por lo que los datos disponibles son difíciles de conseguir y escasos.

Por otro lado, es posible abordar el problema desde el punto de vista computacional. Entre los métodos más utilizados se encuentran los métodos atomísticos que se han usado intensamente en un gran número de sistemas metal /óxido. Los cálculos se concentran principalmente en la determinación del trabajo de separación $\mathrm{W}_{\text {sep }}$. En particular la teoría de la densidad funcional (DFT) [23,24] se utiliza con éxito para en un gran número de casos de decohesión interfacial [3,25-27]. Los cálculos se concentran principalmente en la determinación del trabajo de separación. Por ejemplo en los trabajos de QUI y HECTOR [28,29] se utilizan cálculos de primeros principios para distintos sistemas.

Otro parámetro importante en la descripción de la resistencia interfacial es el comportamiento ante solicitaciones de corte. Esto se debe principalmente a que en sistemas reales la solicitación de las interfaces metal /óxido suelen ser en modo mixto [3,30]. En situaciones tecnológicas, las cargas sobre la interfaz suele ser en tracción pura o modo I, en corte puro o modo II, o cualquier estado intermedio o de mezcla entre ambos modos puros [31,32]. En este contexto, los cálculos a nivel atomístico permiten describir las fuerzas generalizadas que actúan sobre las superficies de los materiales que componen la interfaz en todo el rango de desplazamientos relativos mediante una función potencial generalizada que puede reconstruirse a partir de un número finito de cálculos de energía total [3]. 


\section{MÉTODOS}

La Teoría de la Funcional Densidad (DFT) $[23,24]$ se usa a a través de la implementación en el Viena AbInitio Simulation Package (VASP) [33,34]. Las Interacciones ión-electrón se tratan mediante el método de las ondas planas aumentadas (PAW) [35]. Se considera una expansión en ondas planas con una energía de corte de $500 \mathrm{eV}$. Las interacciones de correlación e intercambio se tratan mediante la aproximación del gradiente generalizado según PERDEW et al. [36]. Las Integraciones en la zona de Brillouin se realizan utilizando una grilla de MONKHOST PACK[37]. Las ocupaciones electrónicas se tratan con el método del tetraedro con correcciones de BLÖCHL [38].

\subsection{Cálculos en volumen}

La Magnetita tiene una estructura de espinela inversa $(F d \overline{3} m)$. Los grados de libertad internos son relajados para obtener el estado fundamental que se muestra en la Figura 1. En esta estructura el oxígeno forma una red fcc mientras que el hierro ocupa los sitios tetraédricos y octaédricos. Estas subredes están acopladas en forma antiferromagnética [39,40]. A lo largo de la dirección 001, las capas atómicas alternan su composición entre $\mathrm{Fe}$ y $\mathrm{FeO}_{2}$. La energía total se calcula en función del volumen y luego se utiliza la ecuación de estado de Birch-Murnaghan para obtener el parámetro de red [41]. Un trabajo similar se lleva a cabo con el hierro teniendo en cuenta su estructura BCC. Los parámetros de red que se encuentran son = $2.842 \AA$ para el hierro BCC ferromagnético y para la magnetita. La grilla de MP se toma de 7x7 7 para la magnetita en volumen y de $15 \times 15 \times 15$ para el hierro BCC, logrando una convergencia de $0.1 \mathrm{meV}$.

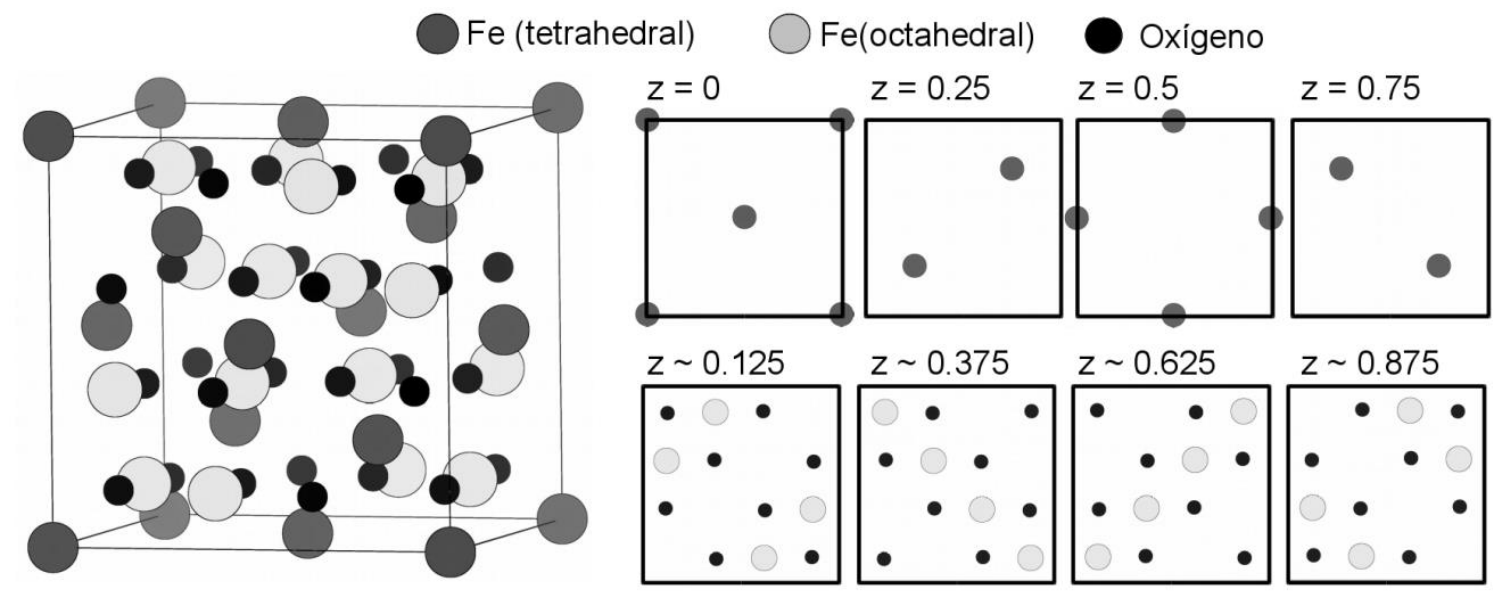

Figura 1: Estructura cristalina de la Magnetita. A la Izquierda, esquema de las posiciones atómicas dentro de la celda unidad. A la derecha se muestra la sucesión de planos [001] con su alternancia en composición.

\subsection{Cálculos de superficie}

DAVENPORT et al. [5] obtuvieron las relaciones de orientación entre un substrato de hierro y la magnetita que crece sobre este. Obtienen que la relación que más ocurre es [100](001)Fe $\|[110](001) \mathrm{Fe}_{3} \mathrm{O}_{4}$. Observan que estas relaciones se deben a que en la dirección [100] del hierro BCC las distancias interatómicas muy similares a las que ocurren en la dirección 110 del óxido. Por lo tanto, en el modelo a construir deben respetarse estas observaciones.

Dado que es necesario reproducir las propiedades de una interfaz en equilibrio con los materiales en volumen, es necesario construir el modelo de interfaz con las láminas de cada material suficientemente gruesas como para que en las capas más internas se reproduzcan las propiedades de los materiales en volumen. Esto se logra estudiando la convergencia de la energía de superficie en función del número de capas atómicas de las laminas superficiales.

El modelo superficial de hierro se construye con una supercelda de simetría tetragonal con un átomo por capa atómica. Las dimensiones en el plano de la superficie son consistentes con el parámetro de red calculado en el volumen. Se introduce un clivaje con una región vacía para prevenir la interacción entre imágenes periódicas. Las pruebas de convergencia muestran que una altura de vacío de $8 \AA$ es suficiente para una lámina de 12 capas atómicas. Luego, siguiendo el método de BOETTGER [42] se calcularon las energías para láminas de 4 a 15 capas atómicas. Se encontró que la energía de superficie está bien convergida para una 
lámina de siete capas atómicas con una tolerancia de $0.03 \mathrm{~J} / \mathrm{m}^{2}$ y con un valor de $2.47 \mathrm{~J} / \mathrm{m}^{2}$, que está en buen acuerdo con otros estudios DFT de hierro BCC [43]. La grilla de Monkhorst-Pack utilizada fue de 15x15x1, mientras que el resto de los parámetros se mantuvieron como en los cálculos de volumen.

Para evaluar la lámina superficial de magnetita, debe tenerse en cuenta que debido a las composiciones alternadas en el plano (001), una lámina superficial estequiométrica de magnetita tendrá necesariamente dos superficies distintas. Para obtener dos superficies equivalentes de composición Fe, es necesario tomar una lámina estequiométrica y agregar una capa atómica de hierros tetraédricos sobre la capa de composición FeO2, reproduciendo la secuencia que se da en el material en volumen. En este caso, la energía de superficie depende del potencial químico de oxígeno y puede calcularse en el límite inferior del rango de estabilidad de la superficie, o límite pobre en oxígeno (O-poor) [1,44]. Las láminas se construyen usando las dimensiones una celda unidad de magnetita para el plano de la interfaz. Las pruebas de convergencia muestran que un vacío de 8 A y un apilamiento de 11 capas atómicas reproducen la energía de superficie en el límite pobre en oxígeno de $1.61 \mathrm{~J} / \mathrm{m}^{2}$ dentro de una tolerancia de $0.01 \mathrm{~J} / \mathrm{m}^{2}$. Para el cálculo se utilizó una grilla de Monkhorst-Pack de 7×7x1, manteniendo el resto de los parámetros como en el cálculo de volumen. No se investiga en este trabajo la lámina de magnetita de terminación $\mathrm{FeO}_{2}$ puesto que se ha mostrado en trabajos anteriores que la interfaz más estable es aquella formada por el óxido con composición $\mathrm{FeO}_{2}$ en su capa atómica terminal.

\subsection{Cálculos de Interfaces}

El modelo de interfaz se construye a partir de las láminas superficiales convergidas en número de capas atómicas como se explicó anteriormente. La supercelda del modelo se muestra en la Figura 2. Se toma la lámina de 11 capas atómicas del óxido con terminación Fe obtenida de las pruebas de convergencia. Sobre ella se apila la lámina de hierro BCC de 7 capas atómicas, a la que se le aplica una rotación de $45^{\circ}$ y se le da una multiplicidad de $2 \times 2$. De esta manera se logra generar la interfaz manteniendo las relaciones de orientación observadas experimentalmente. Debajo de la lámina de óxido y sobre la lámina de hierro se introducen espacios vacíos que en total suman una altura de $8 \AA$, para evitar la interacción entre las superficies libres. De esta manera, la región central de la supercelda es la que emula a la interfaz.

Para el apilamiento entre óxido y metal quedan por determinar la distancia interfacial d y el desplazamiento relativo entre ambas láminas. Analizando el detalle de las vistas de las capas atómicas adyacentes a la interfaz pueden observarse tres posiciones relativas características. En la posición Hollow, el hierro tetraédrico del óxido queda posicionado sobre el sitio octaédrico de la última capa metálica. En la posición Top, el mismo hierro tetraédrico queda directamente sobre un hierro metálico. Por último, en la posición Bridge el hierro tetraédrico queda sobre el punto medio del segmento 100 del metal que une a dos átomos de hierro.

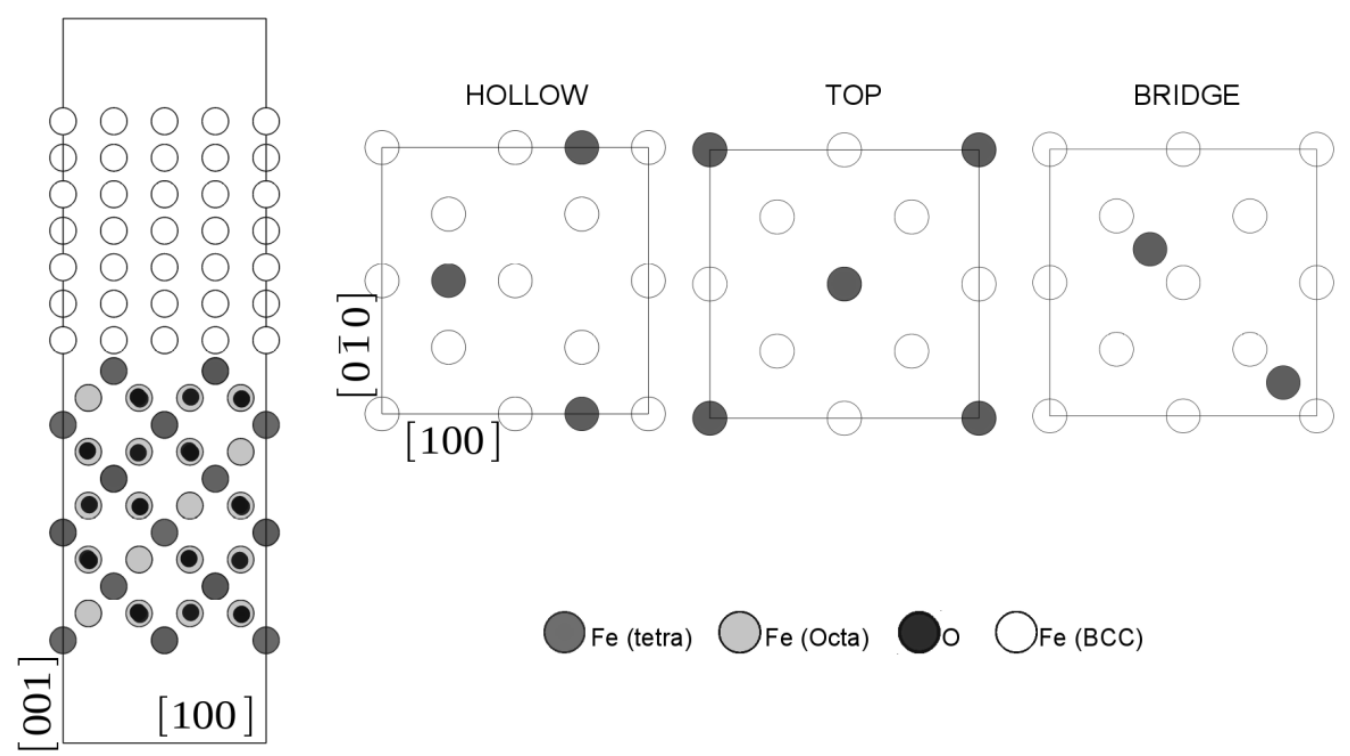

Figura 2: Sobre el borde izquierdo se muestra la supercelda que sirve como modelo de interfaz. Las direcciones indicadas sobre los lados de las superceldas se refieren a la celda unidad del óxido. Las vistas del plano (001) indican los api- 
lamientos Hollow, Top y Bridge desde un punto de vista desde la lámina de óxido. Se muestran la primer capa de metal y las dos primeras capas del óxido adyacentes a la interfaz.

La distancia interfacial es aún un parámetro a determinar. Para hacerlo, para cada una de las posiciones relativas, se arman las superceldas con para un conjunto de valores de d, y se calcula la energía total de cada una de las estructuras. La estructura de equilibrio estará dada por la distancia interfacial que corresponda con el mínimo de energía. De esta manera se logra estudiar el comportamiento de la interfaz ante una solicitación de modo I o tracción pura.

Por último, dado que también es necesario conocer la conducta de la interfaz sobre una solicitación en modo II o de corte, se elabora un plan de cálculo que permita conocer la transición entre las posiciones Hollow, Bridge y Top. Si se toma por ejemplo la posición Hollow, las direcciones 100 y 110 del óxido se comportan como las direcciones principales de la interfaz. Tomando en cuenta la última capa atómica del metal y la capa de hierros tetraédricos en el óxido, la descripción del desplazamiento relativo entre ambas láminas puede describirse a partir de traslaciones de lo que ocurra en el triángulo formado por las posiciones Hollow, Top y Bridge indicado en la Figura 3. Por esta razón, se toman los segmentos entre Hollow y Bridge sobre la dirección 110, y entre Hollow y Top en la dirección 100. Se define una partición de cada uno de estos segmentos de manera de definir una serie discreta de desplazamientos $\mathrm{s}_{100}$ y $\mathrm{s}_{110}$ en las direcciones [100] y [110] respectivamente y en el plano de la interfaz. Para cada uno de estos desplazamientos debe realizarse el mismo barrido en la distancia interfacial $\mathrm{d}$.

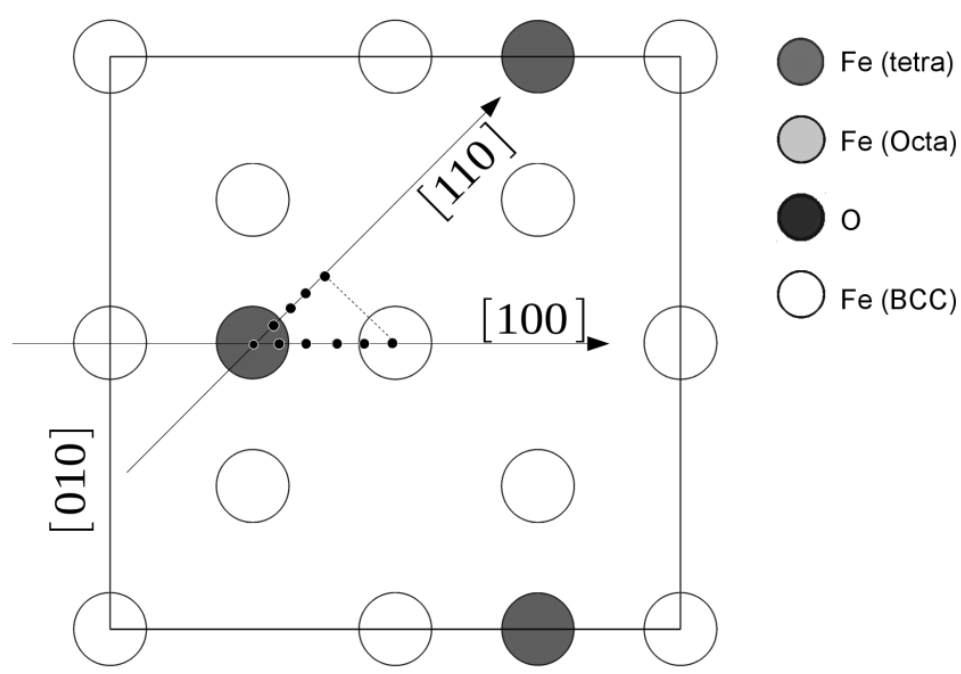

Figura 3: Esquema de la unidad mínima de coordenadas posibles en el plano de la interfaz. Se marcan como ejemplo los puntos con las posiciones

\section{RESULTADOS}

\subsection{Cálculos en modo I - Tracción}

En primer lugar se realizan los cálculos de la energía total de la supercelda en función de la distancia interfacial d para las posiciones características Hollow, Top y Bridge. La distancia interfacial se toma como la altura entre las últimas capas atómicas adyacentes a la interfaz en cada lámina. A partir de las energías totales se define la energía de adhesión

$$
E_{a d}=\frac{1}{A}[E(d)-E(d=\infty)]
$$

en donde A es el área subtendida por la interfaz, E(d) es la energía total calculada para una distancia interfacial dada, y $\mathrm{E}(\mathrm{d}=\infty)$ corresponde a la energía de la supercelda a una separación suficientemente grande como para que la interacción entre láminas sea nula. En la práctica $\mathrm{E}(\mathrm{d}=\infty)$ se toma como la suma de las energías calculadas para las láminas por separado. En todos los casos se toma una grilla de Monkhorst-Pack de 7x7x1 y acoplamiento ferromagnético entre los hierros de las capas atómicas contiguas a la interfaz. La 
energía de adhesión así definida puede ajustarse a una Relación Universal de Energía de Ligadura (UBER) $[45,46]$

$$
E_{a d}(d)=-W_{s e p}\left(1+\frac{d-d_{o}}{l}\right) e^{\frac{d-d_{o}}{l}}
$$

donde $\mathrm{W}_{\text {sep }}$ es el trabajo de separación, $\mathrm{d}_{0}$ es la distancia interfacial de equilibrio y $l$ es un parámetro de escala.

Los resultados del ajuste UBER se muestran en la Tabla 1. Se observa que la configuración Hollow es la interfaz más cohesiva. La distancia interfacial de equilibrio es la menor en este caso. El valor del trabajo de separación para el caso Hollow está en buen acuerdo con los valores medidos mediante ensayos de micro y macro tensión por TOSCAN et al. [22] y NILSONTHI et al. [17] quienes reportan valores de aproximadamente $2 \mathrm{~J} / \mathrm{m}^{2}$ para aceros al carbono. Las energías de fractura para la magnetita obtenidas por LIAO y CARTER [47] mediante un estudio DFT son mayores a $3 \mathrm{~J} / \mathrm{m}^{2}$. Esto indica que el óxido es más resistente que la interfaz en estudio. Según EVANS [48] con esta relación de resistencias, una compresión en la interfaz metal/óxido puede dar lugar al ampollado de la capa de óxido, donde la fisura interfacial puede crecer hasta que el óxido se astille, dejando desprotegido al metal.

Tabla 1: Resultados de ajustes UBER para los apilamientos característicos de la interfaz $\mathrm{Fe} / \mathrm{Fe}_{3} \mathrm{O}_{4}$

\begin{tabular}{l|l|l|l}
\hline INTERFAZ & $\begin{array}{l}\text { TRABAJO DE SEPARA- } \\
\text { CION }\end{array}$ & $\begin{array}{l}\text { DISTANCIA INTERFA- } \\
\text { CIAL DE EQUILIBRIO }\end{array}$ & PARÁMETRO DE ESCALA \\
\hline Hollow & 1.93 & 1.3 & 0.72 \\
\hline Bridge & 1.18 & 1.9 & 0.76 \\
\hline Top & 1.04 & 2.2 & 0.65 \\
\hline
\end{tabular}

\subsection{Cálculos en modo II / corte}

La caracterización del comportamiento de la interfaz en modo I es una parte primordial en la descripción de la resistencia de la interfaz. Sin embargo, sólo con el trabajo de separación no es suficiente para la evaluación de la resistencia bajo una solicitación en modo mixto. La necesidad de este recurso se encuentra en la importancia de la solicitación mixta en los casos de delaminación del óxido superficial. Es necesario, entonces recurrir a un modelo que de cuentas sobre la distribución de tensiones interfaciales con componentes normal y tangencial respecto del plano interfacial. Tal modelo puede encontrarse originalmente en los trabajos de SUN et al. [49] y más tarde en los trabajos de WEI y HUTCHINSON [3,30]. Se trata de un potencial interfacial que puede escribirse de la siguiente manera,

$$
W\left(\delta_{1}, \delta_{2}\right)=\Gamma_{0}\left[-\left(1+\frac{\delta_{2}}{\delta}\right)+f\left(\delta_{1}\right)\left(1+(1+\beta) \frac{\delta_{2}}{\delta}\right)\right] e^{-\frac{\delta_{2}}{\delta}}
$$

donde $\delta_{1}$ y $\delta_{2}$ son los desplazamientos relativos entre los bloques que conforman la interfaz en las direcciones tangencial y normal respectivamente, siempre relativos a la posición de equilibrio. En términos de los parámetros definidos anteriormente,

$$
\begin{aligned}
& \delta_{\mathrm{f}}^{100], \mid 110\rfloor}=s^{\lfloor 100],\lfloor 110\rfloor} \\
& \delta_{2}=d-d_{0}^{\text {Hollow }}
\end{aligned}
$$

siendo $s$ el desplazamiento horizontal desde la posición Hollow hasta alguna posición a considerar, a lo largo de alguna de las direcciones [100] o [110]. Otros parámetros que aparecen son: el trabajo de separación generalizado $\Gamma_{0}$, un nuevo parámetro de escala $\delta$, la función $f\left(\delta_{1}\right)$ y $\beta$, que deberán ser determinados. En primera instancia debe notarse que para una separación normal pura, i.e. $\delta_{1}=0$, la ecuación Erro! Fonte de referência não encontrada. puede reducirse al modelo UBER de la ecuación Erro! Fonte de referência não encontrada., ya que dicho modelo ha demostrado ser representativo de cálculos atomísticos en un gran número de interfaces. Por lo tanto puede imponerse que $f\left(\delta_{1}=0\right)=0$ y entonces debe ser que $\Gamma_{0}=\mathrm{W}_{\text {sep }}{ }^{\text {Hollow }} \mathrm{y}$ $\delta=l^{\text {Hollow }}$. Queda entonces determinar la forma que toma $f\left(\delta_{1}\right)$ y $\beta$. Para ello se trabaja sobre el esquema de 
cálculo presentado en la Figura 4, tomando particiones de los segmentos Hollow - Top en la dirección [100] y Hollow-Bridge en la dirección [100], barriendo en las coordenadas $\delta_{1}$ en cada dirección, y para cada valor de $\delta_{1}$ se calculan las energías con una serie de valores de $\delta_{2}$.

El potencial interfacial de la ecuación Erro! Fonte de referência não encontrada. puede linealizarse de la siguiente forma

$$
\begin{aligned}
& \operatorname{LINEAL}_{\left(\frac{\delta_{2}}{\delta}\right)}= \\
& \frac{E\left(\delta_{1}, \delta_{2}\right)-E\left(\delta_{2} \rightarrow \infty\right)}{\Gamma_{0}} \exp \left(\frac{\delta_{2}}{\delta}\right)=\left(-1+f\left(\delta_{1}\right)\right)+\left[-1+f\left(\delta_{1}\right)(1+\beta)\right] \frac{\delta_{2}}{\delta}
\end{aligned}
$$

de manera que para cada serie de energías $\mathrm{E}\left(\delta_{1}, \delta_{2}\right)$ con $\delta_{1}$ fijo, $f\left(\delta_{1}\right)$ y $\beta$ resultan de un ajuste lineal a la función LINEAL así definida. En los trabajos de Hutchinson y Wei se toma una alternativa algo distinta, donde se plantean una serie de cálculos de energía total en algunos sitios característicos, primero con $\delta_{1}=0 \mathrm{y}$ luego con $\partial \mathrm{W} / \partial \delta_{2}=0$, para obtener por reemplazo directo los parámetros incógnita. Esta aproximación es algo más sofisticada a la que se presenta aquí, pero requiere conocer con cierta precisión el punto de mínima energía para cada $\delta_{1}$, lo que puede ser tan costoso como el planteo que conlleva la resolución por ajuste lineal de la ecuación Erro! Fonte de referência não encontrada..

El abanico de rectas que se obtiene para cada dirección se observa en la Figura 5. $\beta$ resulta ser una constante aproximadamente igual a 1.45 para todos los puntos de ambas direcciones, lo cual es consistente con el planteo inicial y con lo reportado en trabajos de otros autores. La función $f\left(\delta_{1}\right)$ se ilustra en la Figura 6.

\section{DISCUSIÓN}

Con las determinaciones de los parámetros intervinientes en la Ecuación Erro! Fonte de referência não encontrada. se logra tener una descripción completa del potencial interfacial. La ventaja de este conocimiento es que a partir del potencial es posible tener la ley de tensiones entre las superficies interactuantes en una solicitación de carácter general,

$$
T_{1}=\frac{\partial W}{\partial \delta_{1}} ; \quad T_{2}=\frac{\partial W}{\partial \delta_{2}}
$$

donde $\mathrm{T}_{1}$ y $\mathrm{T}_{2}$ son las las fuerzas por unidad de área que actúan sobre las superficies que conforman la interfaz en las direcciones tangencial y normal respectivamente. Esta ley de fuerzas puede ser usada como entrada en modelos de escala superior de la respuesta de la interfaz ante solicitaciones más generales y en escala de orden superior. TVERGAARD y HUTCHINSON [50] realizaron una serie de estudios donde obtienen leyes fenomenológicas para las relaciones tracción-desplazamiento para luego aplicar en un modelo de elementos finitos incluyendo la influencia de la plasticidad de uno de los componentes de la interfaz. Más recientemente WEI y HUTCHINSON [30] implementan el mismo método, pero obteniendo las tracciones y el potencial interfacial a partir de cálculos DFT como los aquí presentados, para aplicar en un modelo de elementos finitos en sistemas metal / óxido. 


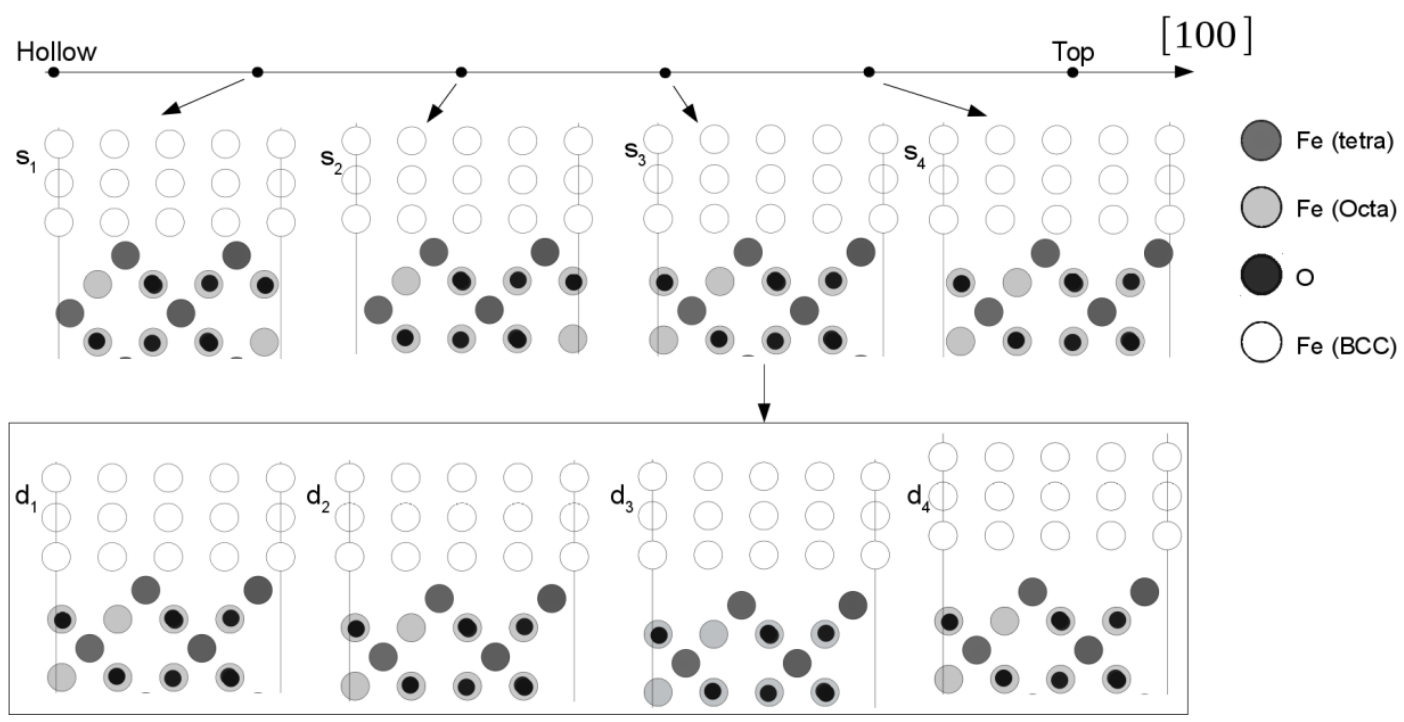

Figura 4: Esquema de cálculo para la resolución de los parámetros $f\left(\delta_{1}\right)$ y $\beta$ del potencial interfacial en la ecuación Erro! Fonte de referência não encontrada.. En el ejemplo del esquema se toma una partición del segmento Hollow - Top sobre la dirección [100]. para cada valor de s se evalúan varios valores de d.
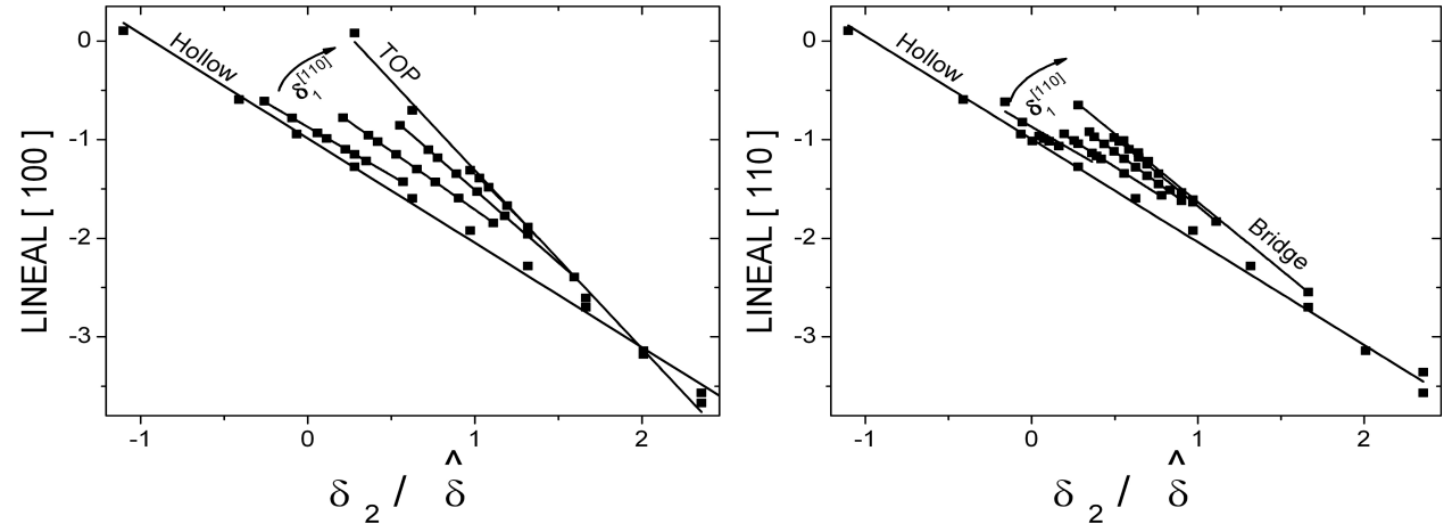

Figura 5: Función LINEAL detallada en la ecuación Erro! Fonte de referência não encontrada..Los puntos corresponden a los cálculos, las líneas sólidas corresponden al ajuste lineal, siendo una recta para cada serie de $\delta_{1}$.

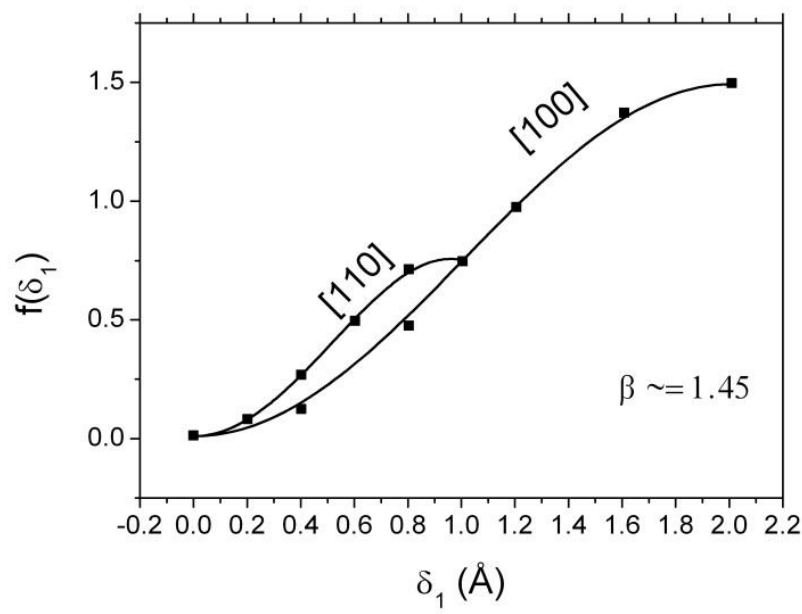

Figura 6: Función $f\left(\delta_{1}\right)$ en cada dirección. Los puntos corresponden con los ajustes de los abanicos de curvas de la Figura 5. Las líneas sólidas corresponden a un ajuste a estos datos calculados usando series de funciones trigonométricas. 


\section{CONCLUSIONES}

En el presente trabajo se utiliza un modelo atomístico DFT para evaluar las propiedades de la interfaz $\mathrm{Fe} /$ $\mathrm{Fe}_{3} \mathrm{O}_{4}$ ante solicitaciones de corte y detracción. Se revisó la construcción de los bloques superficiales que se utilizarían luego para la construcción del modelo interfacial. Se comparó la adhesividad de los apilamientos característicos de la interfaz $\mathrm{Fe}_{3} \mathrm{O}_{4} / \mathrm{Fe}(\mathrm{BCC})$ en el caso donde el hierro tiene una composición Fe en la capa atómica terminal, y utilizando el trabajo de separación como el parámetro principal. Siendo el apilamiento Hollow el de mayor adhesividad, se lo utiliza como punto de partida para evaluar el comportamiento del sistema ante solicitaciones de corte en las direcciones principales de la interfaz. Se obtuvieron los parámetros necesarios para completar la descripción del potencial interfacial a partir de los modelos disponibles en la literatura. Este potencial interfacial puede utilizarse como entrada de un modelo de escala superior para el estudio completo de los sistemas metal / óxido y los modos de falla característicos.

\section{AGRADECIMIENTOS}

Este trabajo se realizó en la Gerencia de Materiales CAC-CNEA, y fué parcialmente financiado por la ANPCyT a través del subsidio PICT-2011-1861, y por la Universidad Nacional de San Martín a través del proyecto C070 (2013-2014). Los cálculos se realizaron en el Centro de Cómputos de Altas Prestaciones del Centro Atómico Constituyentes, Comisión Nacional de Energía Atómica, así como en las facilidades exclusivas de la División de Aleaciones Especiales de la Gerencia Materiales, CAC-CNEA. Las visualizaciones de las estructuras se realizan con VESTA [51].

\section{BIBLIOGRAFÍA}

[1] FORTI , M.D., ALONSO, P.R., GARGANO, P.H., et al.,"A DFT study of atomic structure and adhesion at the $\mathrm{Fe}(\mathrm{BCC}) / \mathrm{Fe} 3 \mathrm{O} 4$ interfaces”, Surface Science, v. 647, pp. 55-65 2016.

[2] FORTI , M., ALONSO , P., GARGANO , P., et al.,"Adhesion Energy of the Fe(BCC)/Magnetite Interface within the DFT Approach", Procedia Materials Science, v. 8, pp. 1066-1072 2015.

[3] JIANG , Y., WEI , Y., SMITH , J.R., et al.,"First principles based predictions of the toughness of a metal/oxide interface", International Journal of Materials Research (Formerly Zeitschrift Fuer Metallkunde), v. 101, n. 1, pp. 8-15 2010.

[4] DAVENPORT , A.J., OBLONSKY , L.J., RYAN , M.P., et al.,"The Structure of the Passive Film That Forms on Iron in Aqueous Environments", Journal of The Electrochemical Society, v. 147, n. 6, p. 2162 2000.

[5] TONEY , M., DAVENPORT , A.J., OBLONSKY, L., et al.,"Atomic Structure of the Passive Oxide Film Formed on Iron", Physical Review Letters, v. 79, n. 21, pp. 4282-4285 1997.

[6] YI , W., MOBERLYCHAN , W., NARAYANAMURTI , V., et al., "Characterization of spinel iron-oxide nanocrystals grown on Fe whiskers”, Journal of Applied Physics, v. 95, n. 11, p. 71362004.

[7] CORNELL , R.M., SCHWERTMANN, U. The Iron Oxides, Second Edition, WILEY-VCH VerlagGmbH\& Co., , Weinheim, 2003.

[8] REGAZZONI , A.E., URRUTIA , G.A., BLESA , M.A., et al.,"Some observations on the composition and morphology of synthetic magnetites obtained by different routes", Journal of Inorganic and Nuclear Chemistry, v. 43, n. 7, pp. 1489-1493 1981.

[9] BRUYERE, V., Disolución de Magnetita por transferencia de iones de Fe(III), Tesis de D. Sc. , Universidad de Buenos Aires, 1987.

[10] WANG , X.C., MAO , Z.W., YANG , Q.,"Research on High Pressure Abrasive Water Jet for Cold Rolling Descaling”, Advanced Materials Research, v. 572, pp. 31-36 2012.

[11] VOGES , K., MUETH , A., STREET , S.M., et al.,"Eco Pickled Surface : an Environmentally Advantageous Alternative To Conventional Acid Pickling", In: The Iron \& Steel Technology Conference And Exposition, AISTech 2007, 2007.

[12] GILLSTRÖM , P., JARL, M.,"Replacement of pickling with shot blasting for wire rod preparation", Scandinavian Journal of Metallurgy, v. 33, pp. 269-278 2004.

[13] KRZYZANOWSKI , M., YANG, W., SELLARS , C.M., BEYNON , J.H.,"Analysis of mechanical descaling: Experimental and modelling approach", Materials Science and Technology, v. 19, n. 1, pp. 1091162003.

[14] SCHÜTZE, M.,"Modelling oxide scale fracture”, Materials at High Temperatures, v. 22, n. 1, pp. 
$147-1542005$.

[15] SCHÜTZE , M., TORTORELLI , P.F., WRIGHT , I.G., "Development of a Comprehensive Oxide Scale Failure Diagram”, Oxidation of Metals, v. 73, n. 3-4, pp. 389-418 2009.

[16] GUO , X., SHANG , F.,"Shear strength and sliding behavior of Ni/Al2O3 interfaces: A first-principle study", Journal of Materials Research, v. 27, n. 9, pp. 1237-1244 2012.

[17] NILSONTHI , T., CHANDRA-AMBHORN , S., WOUTERS , Y., GALERIE , A., “Adhesion of thermal oxide scales on hot-rolled conventional and recycled steels", Oxidation of Metals, v. 79, n. 3-4, pp. 325-335 2013.

[18] ZHANG , W., SMITH , J.R., EVANS, A.G. "The connection between ab initio calculations and interface adhesion measurements on metal/oxide systems: Ni/Al2O3 and Cu/Al2O3", Acta Materialia, v. 50, n. 15, pp. 3803-3816 2002.

[19] MIYOSHI , K., POHLCHUCK , B., WHITTLE , N.C., et al., Properties data for adhesion and surface chemistry of. Aluminum, NASA Technical Publication ,1998

[20] MOUGIN , J., DUPEUX , M., GALERIE, A., et al., "Inverted blister test to measure adhesion energy of thermal oxide scales on metals or alloys", Materials Science and Technology, v. 18, n. 10, pp. 1217-1220 2002.

[21] CHANDRA-AMBHORN, S., WOUTERS ,Y., ANTONI, L., et al.,"Adhesion behaviour of thermal oxide scales grown on ferritic stainless steels proposed as interconnects in SOFCs", In: Proceedings - Electrochemical Society, 2005.

[22] TOSCAN , F., ANTONI , L., WOUTERS, Y., et al., "Spalling and Mechanical Aspects-Oxidation Kinetics and Scale Spallation of Iron-Chromium Alloys with Different Titanium Contents", Materials Science Forum, v. 461, pp. 705-712 2004.

[23] HOHENBERG , P., KOHN , W., "Inhomogeneous electron gas”, Phys. Rev, v. 136, n. 3B, pp. B864B871 1964.

[24] KOHN , W., SHAM , L.J. "'Self-Consistent Equations Including Exchange and Correlation Effects”, Physical Review, v. 140, n. 4A, pp. A1133-A1138, 1965.

[25] EREMEEV, S.V., SCHMAUDER, S., HOCKER, S., et al., “Ab-initio investigation of $\mathrm{Ni}(\mathrm{Fe}) / \mathrm{ZrO} 2(001)$ and Ni-Fe/ZrO2(001) interfaces”, Surface Science, v. 603, n. 14, pp. 2218-2225 2009.

[26] MATSUNAKA, D., SHIBUTANI , Y., "Electronic states and adhesion properties at metal/MgO incoherent interfaces: First-principles calculations", Physical Review B - Condensed Matter and Materials Physics, v. 77, n. 16, pp. 1-62008.

[27] UDAGAWA , Y., YAMAGUCHI , M., ABE , H., et al.,"Ab initio study on plane defects in zirconiumhydrogen solid solution and zirconium hydride”, Acta Materialia, v. 58, n. 11, pp. 3927-3938 2010.

[28] QI , Y., HECTOR , L.G., “Adhesion and adhesive transfer at aluminum/diamond interfaces: A firstprinciples study”, Physical Review B, v. 69, n. 23, p. 2354012004.

[29] QI , Y., HECTOR , L.G.,"Hydrogen effect on adhesion and adhesive transfer at aluminum/diamond interfaces", Physical Review B, v. 68, n. 20, p. 2014032003.

[30] WEI , Y., HUTCHINSON , J.W., "Toughness of Ni/A12O3 interfaces as dependent on micron-scale plasticity and atomistic-scale separation", Philosophical Magazine, v. 88, n. 30, pp. 3841-3859 2008.

[31] HUTCHINSON , J.W., SUO , Z., "Mixed Mode Cracking in Layered Materials", Advances in Applied Mechanics, v. 29, n. C, pp. 63-191 1991.

[32] EVANS , A.G., HUTCHINSON , J.W.,"The mechanics of coating delamination in thermal gradients", Surface and Coatings Technology, v. 201, n. 18, pp. 7905-7916 2007.

[33] HAFNER , J., “Ab-initio simulations of materials using VASP: Density-functional theory and beyond”, Journal of Computational Chemistry, v. 29, n. 13, pp. 2044-2078 2008.

[34] HAFNER, J.,"Materials simulations using VASP — a quantum perspective to materials science" , Computer Physics Communications, v. 177, n. 1-2, pp. 6-13 2007.

[35] VANDERBILT , D., "Soft self-consistent pseudopotentials in a generalized eigenvalue formalism", Physical Review B, v. 41, n. 11, pp. 7892-7895 1990.

[36] PERDEW , J., BURKE , K., ERNZERHOF , M., "Generalized Gradient Approximation Made Simple", Physical Review Letters, v. 77, n. 18, pp. 3865-3868 1996.

[37] MONKHORST , H.H.J., PACK , J.J.D., “Special points for brillouin-zone integrations”, Physical Review $B$, v. 13, n. 12, pp. 5188-5192 1976. 
[38] BLÖCHL , P.E., JEPSEN , O., ANDERSEN , O.K.,"Improved tetrahedron method for brillouin-zone integrations", Physical Review B, v. 49, n. 23, pp. 16223-16233 1994.

[39] ROWAN , A., PATTERSON , C., GASPAROV , L.,"Hybrid density functional theory applied to magnetite: Crystal structure, charge order, and phonons", Physical Review B, v. 79, n. 20, pp. 1-18 2009.

[40] ZHANG , Z., SATPATHY, S.,"Electron states, magnetism, and the Verwey transition in magnetite", Physical Review B, v. 44, n. 24, pp. 13319-13331 1991.

[41] BIRCH , F.,"The Effect of Pressure Upon the Elastic Parameters of Isotropic Solids, According to Murnaghan's Theory of Finite Strain”, Journal of Applied Physics, v. 9, n. 4, p. 2791938.

[42] BOETTGER, J.,"Nonconvergence of surface energies obtained from thin-film calculations", Physical Review B, v. 49, n. 23, pp. 798-800 1994.

[43] BŁOŃSKI , P., KIEJNA , A.,"Structural, electronic, and magnetic properties of bcc iron surfaces", Surface Science, v. 601, n. 1, pp. 123-133 2007.

[44] ROGAL, J.,Stability, Composition and Function of Palladium Surfaces in Oxidizing Environments : A First-Principles Statistical Mechanics Approach, Tesis de D. Sc. , Freien Universitat Berlin, 2006.

[45] BANERJEA , A., SMITH , J.R., “Origins of the universal binding-energy relation”, Physical Review B, v. 37, n. 12, p. 66321988.

[46] EVANS , A.G., HUTCHINSON , J.W., WEI , Y.,"Interface adhesion: effects of plasticity and segregation”, Acta Materialia, v. 47, n. 15, pp. 4093-4113 1999.

[47] LIAO , P., CARTER , E., “Ab initio DFT + U predictions of tensile properties of iron oxides”, Journal of Materials Chemistry, v. 20, n. 32, 2010.

[48] EVANS , H.,"Spallation of oxide from stainless steel AGR nuclear fuel cladding: mechanisms and consequences", Materials Science and Technology, v. 4, pp. 415-420, 1988.

[49] SUN , Y., BELTZ , G., RICE , J.,"Estimates from atomic models of tension-shear coupling in dislocation nucleation from a crack tip", Materials Science and Engineering: A, v. 170, n. 1-2, pp. 67-85 1993.

[50] TVERGAARD , V., HUTCHINSON , J.W.,"The influence of plasticity on mixed mode interface toughness", Journal of the Mechanics and Physics of Solids, v. 41, n. 6, pp. 1119-1135 1993.

[51] MOMMA , K., IZUMI , F., "VESTA : a three-dimensional visualization system for electronic and structural analysis”, Journal of Applied Crystallography, v. 41, n. 3, pp. 653-658 2008. 